\title{
ON CARTAN SUBALGEBRAS AND CARTAN SUBSPACES OF NONSYMMETRIC PAIRS OF LIE ALGEBRAS
}

\author{
BORIS ŠIROLA \\ (Communicated by Gail R. Letzter)
}

\begin{abstract}
Let $\left(\mathfrak{g}, \mathfrak{g}_{1}\right)$ be a pair of Lie algebras, defined over a field of characteristic zero, where $\mathfrak{g}$ is semisimple and $\mathfrak{g}_{1}$ is a subalgebra reductive in $\mathfrak{g}$. We prove a result giving a necessary and sufficient technical condition so that the following holds: (Q1) For any Cartan subalgebra $\mathfrak{h}_{1} \subseteq \mathfrak{g}_{1}$ there exists a unique Cartan subalgebra $\mathfrak{h} \subseteq \mathfrak{g}$ containing $\mathfrak{h}_{1}$. Next we study a class of pairs $\left(\mathfrak{g}, \mathfrak{g}_{1}\right)$, satisfying $(\mathbf{Q 1})$, which we call Cartan pairs. For such pairs and the corresponding Cartan subspaces, we prove some useful results that are classical for symmetric pairs. Thus we extend a part of the previous research on Cartan subspaces done by Dixmier, Lepowsky and McCollum.
\end{abstract}

\section{INTRODUCTION}

Throughout this paper $\mathbb{K}$ always denotes a field of characteristic zero, and by $\overline{\mathbb{K}}$ we denote its fixed algebraic closure. All Lie algebras are finite dimensional; and, unless specified otherwise, they are defined over $\mathbb{K}$. If $\mathfrak{g}$ is a reductive Lie algebra and $\mathfrak{h}$ is its split Cartan subalgebra, by $\Delta(\mathfrak{g}, \mathfrak{h})$ we denote the root system of $\mathfrak{g}$ with respect to $\mathfrak{h}$. For a root $\phi$, by $X_{\phi}$ we denote a nonzero vector from the corresponding root subspace $\mathfrak{g}_{\phi}$. Given any $\mathbb{K}$-Lie algebra $\mathfrak{g}$, we define $\overline{\mathfrak{g}}=\mathfrak{g} \otimes \overline{\mathbb{K}}$. Also by $B_{\mathfrak{g}}$ we denote the Killing form of $\mathfrak{g}$.

Let $\mathfrak{g}$ be a semisimple Lie algebra. Let $\mathfrak{g}_{1}$ be a proper subalgebra which is reductive in $\mathfrak{g}$. Given the pair $\left(\mathfrak{g}, \mathfrak{g}_{1}\right)$, we can ask whether the following condition is satisfied:

(Q1) For any Cartan subalgebra $\mathfrak{h}_{1} \subseteq \mathfrak{g}_{1}$ there exists a unique Cartan subalgebra $\mathfrak{h} \subseteq \mathfrak{g}$ containing $\mathfrak{h}_{1}$.

It turns out that although $(\mathbf{Q} \mathbf{1})$ is a rather strong condition on pairs $\left(\mathfrak{g}, \mathfrak{g}_{1}\right)$, there are a number of interesting pairs that satisfy it. For more details about special pairs of that kind we refer to $\mathrm{BK}$, and in particular to Theorem 5.3 there; see also [LS], V1 and V2, which partly motivated the research of Brylinski and Kostant. For another subclass of pairs satisfying $(\mathbf{Q 1})$, see $[\check{S} 3$.

As further motivation, perhaps it might be instructive to recall the following. For the moment let the base field $\mathbb{K}$ be algebraically closed. Suppose that a pair $\left(\mathfrak{g}, \mathfrak{g}_{1}\right)$

Received by the editors December 2, 2010 and, in revised form, September 16, 2011 and October 11, 2011.

2010 Mathematics Subject Classification. Primary 17B05; Secondary 17B20, 17B22.

Key words and phrases. Pair of Lie algebras, semisimple Lie algebra, reductive subalgebra, Cartan subalgebra, Cartan subspace, Cartan pair.

The author was supported in part by the Ministry of Science, Education and Sports, Republic of Croatia, Grant No. 900-194134. 
satisfies (Q1), and let $\left(\mathfrak{h}, \mathfrak{h}_{1}\right)$ be the corresponding pair of Cartan subalgebras. If one would like to study embedding and/or restricting of certain representations, it is helpful to understand how the root systems $\Delta=\Delta(\mathfrak{g}, \mathfrak{h})$ and $\Delta_{1}=\Delta\left(\mathfrak{g}_{1}, \mathfrak{h}_{1}\right)$ are related to each other. In particular we have to know how a root vector $X_{\psi}, \psi \in \Delta_{1}$, can be written as a linear combination of root vectors $X_{\varphi}, \varphi \in \Delta$; see, e.g., LS, Sect. 2.3]. From the point of view of representation theory it is also useful to find various pairs of compatible Borel (or parabolic) subalgebras $\left(\mathfrak{b}, \mathfrak{b}_{1}\right)$. Here, a pair $\left(\mathfrak{b}, \mathfrak{b}_{1}\right)$ is compatible if $\mathfrak{h}_{1} \subseteq \mathfrak{b}_{1} \subseteq \mathfrak{g}_{1}, \mathfrak{h} \subseteq \mathfrak{b} \subseteq \mathfrak{g}$ and $\mathfrak{b}_{1} \subseteq \mathfrak{b}$. Notice also that in a number of cases, for $\mathfrak{b}_{1}$ chosen, the corresponding $\mathfrak{b}$ is unique; see [ᄃ5].

In [ک̌2 2 , Thm. 3.5] we gave a sufficient technical condition, (b) of Theorem 0.1 . that can always be easily checked for a concrete pair $\left(\mathfrak{g}, \mathfrak{g}_{1}\right)$ in order to see whether it satisfies (Q1). The first goal of this paper is to present a theorem which shows that in the case when the base field is algebraically closed, the mentioned condition is in fact necessary.

Theorem 0.1. Let $\mathfrak{g}$ be a semisimple $\mathbb{K}$-Lie algebra, and let $\mathfrak{g}_{1}$ be subalgebra reductive in it. Let $\mathfrak{h}_{1}$ be any Cartan subalgebra of $\mathfrak{g}_{1}$. Consider the following three statements:

(a) There exists $\vartheta \in \mathfrak{h}_{1}$ which is regular in $\mathfrak{g}$.

(b) Let $\mathfrak{c}_{1}$ and $\mathfrak{c}$ be any Cartan subalgebras of $\overline{\mathfrak{g}_{1}}$ and $\overline{\mathfrak{g}}$, respectively, such that $\mathfrak{c}_{1} \subseteq \mathfrak{c}$. Then, for every root $\phi \in \Delta(\overline{\mathfrak{g}}, \mathfrak{c})$, the restriction

$$
\phi_{\mid \mathfrak{c}_{1}} \neq 0 .
$$

(c) There exists a unique Cartan subalgebra $\mathfrak{h}$ of $\mathfrak{g}$ such that $\mathfrak{h}_{1} \subseteq \mathfrak{h}$.

Then (a) is equivalent to (b), and (b) implies (c). Furthermore, if $\mathbb{K}=\overline{\mathbb{K}}$, then all three statements are mutually equivalent, and in that case $\mathfrak{h}$ is equal to the centralizer $C_{\mathfrak{g}}\left(\mathfrak{h}_{1}\right)$.

Our proof of the theorem strongly relies on a result, Proposition 1.3. which is interesting in its own right. It points at specific semisimple elements of an arbitrary semisimple Lie algebra. As a useful consequence of this theorem we have Corollary 1.5, which is an improvement of [ک̌ 3 , Thm. 3].

The notion of Cartan subspace for real and complex semisimple symmetric Lie algebras is both crucial and standard. A generalization for semisimple symmetric Lie algebras over an arbitrary field of characteristic zero is due to Dixmier [D, Ch. I, §1.13]. A further generalization and certain interesting results for not necessarily semisimple Lie algebras have been obtained in the nice paper [LM] by Lepowsky and McCollum. The second goal of this paper is to present some evidence that the standard definition of a Cartan subspace makes sense for an interesting class of semisimple nonsymmetric pairs of Lie algebras: that is, pairs $\left(\mathfrak{g}, \mathfrak{g}_{1}\right)$ where $\mathfrak{g}$ is semisimple, $\mathfrak{g}_{1} \subseteq \mathfrak{g}$ is reductive in $\mathfrak{g}$, and the restriction of the Killing form $B_{\mathfrak{g}}$ to $\mathfrak{g}_{1}$ is nondegenerate, but there is no involutive automorphism $\theta$ of $\mathfrak{g}$ such that the fixed point algebra $\mathfrak{g}^{\theta}$ is equal to $\mathfrak{g}_{1}$. More precisely, we consider certain such pairs that we call Cartan pairs; see Definition 2.6. In particular, semisimple symmetric pairs are Cartan pairs; see Proposition 2.13. For Cartan pairs and the corresponding Cartan subspaces, we proved several useful results that are classical for symmetric pairs; see Propositions 2.7 and 2.8. Thus we have improved significantly our previous research [Ś 1 . Let us emphasize that the main motivation for the material presented 
in Section 2 is the celebrated example of complex Lie algebras of type $\left(B_{3}, G_{2}\right)$; see [BK], [LS], and Example 2.14 here. This pair is a nonsymmetric Cartan pair (of type I). Other such pairs are some of those studied in [ัS3].

\section{Proof of Theorem 0.1}

Let $\mathfrak{g}$ be a semisimple Lie algebra. Recall that a subset of nonzero elements $\mathcal{T}=\{\mathrm{f}, \mathrm{h}, \mathrm{e}\} \subseteq \mathfrak{g}$ is called a standard triple if we have $[\mathrm{h}, \mathrm{e}]=2 \mathrm{e},[\mathrm{h}, \mathrm{f}]=-2 \mathrm{f}$ and $[\mathrm{e}, \mathrm{f}]=\mathrm{h}$. Following Kostant $\left[\mathrm{Ks}\right.$, a Lie subalgebra $\operatorname{span}_{\mathbb{K}} \mathcal{T}$ of $\mathfrak{g}$ is called a TDS. Recall also the following two standard results; see [B, Ch. I, §6.3].

Lemma 1.1. Let $\mathfrak{r}$ be a semisimple subalgebra of $\mathfrak{g l}(V)$, where $V$ is a finitedimensional $\mathbb{K}$-vector space, and $X \in \mathfrak{r}$. Then $X$ is a semisimple (resp. nilpotent) element of $\mathfrak{r}$ if and only if $X$ is semisimple (resp. nilpotent) as a linear endomorphism of $V$.

Lemma 1.2. Suppose that $\mathfrak{r}$ and $\mathfrak{g}$ are semisimple Lie algebras, and $\rho: \mathfrak{r} \rightarrow \mathfrak{g}$ is a homomorphism. If $X \in \mathfrak{r}$ is semisimple (resp. nilpotent), then $\rho(X)$ is semisimple (resp. nilpotent) as an element of $\mathfrak{g}$.

In general, having a (reductive) Lie algebra $\mathfrak{g}$, it is useful to have explicit constructions of various semisimple, and nilpotent, elements of $\mathfrak{g}$. As an auxiliary result for proving Theorem 0.1 we have this easy proposition.

Proposition 1.3. Let $\mathfrak{g}$ be a semisimple Lie algebra. Suppose that an element $\mathrm{e} \in \mathfrak{g}$ is nilpotent and $\{\mathrm{f}, \mathrm{h}, \mathrm{e}\}$ is a standard triple. For arbitrary nonzero $a, b \in \mathbb{K}$ define

$$
W=W_{a, b}=a \mathrm{e}+b \mathrm{f} .
$$

Then $W$ is semisimple in $\mathfrak{g}$.

Proof. Consider $\mathfrak{r}=\mathfrak{s l}(2, \mathbb{K})$ as a subalgebra of $\mathfrak{g l}(V)$, where $V=\mathbb{K}^{2}$. Define a homomorphism

$$
\rho: \mathfrak{r} \rightarrow \mathfrak{g}, \quad \rho\left(\left[\begin{array}{cc}
d & a \\
b & -d
\end{array}\right]\right)=d \mathbf{h}+a \mathrm{e}+b \mathfrak{f} .
$$

Take an element $w=\left[\begin{array}{ll}0 & a \\ b & 0\end{array}\right]$, where $a$ and $b$ are nonzero. The characteristic polynomial of $w \in$ End $V$ is $k_{w}(T)=T^{2}-a b$. As $k_{w}(T)$ has two distinct eigenvalues in $\overline{\mathbb{K}}, w$ is semisimple as a linear endomorphism of $V$. By Lemma 1.1, $w$ is semisimple in $\mathfrak{r}$. Finally, by Lemma 1.2, $W=\rho(w)$ is semisimple in $\mathfrak{g}$.

For later applications, we formulate a special case of the above proposition as a corollary.

Corollary 1.4. Let $\mathfrak{g}$ be a semisimple Lie algebra, and suppose that it has a split Cartan subalgebra $\mathfrak{h}$. Then for an arbitrary root $\phi \in \Delta(\mathfrak{g}, \mathfrak{h})$ and nonzero $X_{0} \in \mathfrak{g}_{\phi}$ and $Y_{0} \in \mathfrak{g}_{-\phi}$, the element

$$
W_{0}=X_{0}-Y_{0}
$$

is semisimple in $\mathfrak{g}$.

Proof of Theorem 0.1 . Supposing that $\mathbb{K}$ is algebraically closed, we only have to show that $(\mathrm{c}) \Rightarrow(\mathrm{b})$; for the remaining implications, see $\underline{\mathrm{S} 2}$. With no loss of generality we can assume that $\mathfrak{g}$ is simple. Suppose also that (b) does not hold. This means that we can find two Cartan subalgebras $\mathfrak{c}_{1} \subseteq \mathfrak{c}$ of $\mathfrak{g}_{1} \subseteq \mathfrak{g}$, respectively, so 
that we have a root $\phi_{0} \in \Delta(\mathfrak{g}, \mathfrak{c})$ satisfying $\phi_{0 \mid \mathfrak{c}_{1}}=0$. Let $\mathfrak{g}_{\phi_{0}}=\mathbb{K} \mathrm{x}_{0}$. Let $\mathrm{h}_{0} \in \mathfrak{c}$ be such that $\phi_{0}\left(\mathrm{~h}_{0}\right)=2$. Let $\mathrm{y}_{0} \in \mathfrak{g}_{-\phi_{0}}$ be the unique element such that $\left[\mathrm{x}_{0}, \mathrm{y}_{0}\right]=\mathrm{h}_{0}$. Then of course

$$
\mathfrak{s}=\operatorname{span}_{\mathbb{K}}\left\{\mathrm{y}_{0}, \mathrm{~h}_{0}, \mathrm{x}_{0}\right\}
$$

is a TDS. Define an element

$$
W_{0}=\mathrm{x}_{0}-\mathrm{y}_{0} .
$$

It follows at once that $\left[\mathfrak{c}_{1}, W_{0}\right]=0$ and

$$
\left[\mathrm{h}_{0}, W_{0}\right]=2\left(\mathrm{x}_{0}+\mathrm{y}_{0}\right) .
$$

By Corollary 1.4, we know that $W_{0}$ is semisimple in $\mathfrak{g}$. Define

$$
\mathfrak{a}^{\prime}=\mathfrak{c}_{1} \oplus \mathbb{K} W_{0} .
$$

Clearly, $\mathfrak{a}^{\prime}$ is a commutative subalgebra of $\mathfrak{g}$ consisting of semisimple elements. Thus there exists a Cartan subalgebra $\mathfrak{c}^{\prime}$ of $\mathfrak{g}$ such that $\mathfrak{a}^{\prime} \subseteq \mathfrak{c}^{\prime}$. We claim that $\mathfrak{c} \neq \mathfrak{c}^{\prime}$. If not, then in particular we would have both $W_{0}$ and $\mathrm{h}_{0}$ in $\mathfrak{c}$, and therefore $\left[W_{0}, \mathrm{~h}_{0}\right]=0$. But this is in contradiction with (11). Thus we have proved that (c) does not hold, and so the theorem follows.

Suppose now that we have Lie algebras $\mathfrak{s} \subseteq \mathfrak{g}_{1} \subseteq \mathfrak{g}$, defined over an algebraically closed field $\mathbb{K}$, so that $\mathfrak{g}_{1}$ and $\mathfrak{g}$ are semisimple and $\mathfrak{s}$ is reductive in $\mathfrak{g}_{1}$. (Of course, then $\mathfrak{s}$ is also reductive in $\mathfrak{g}$; see, e.g., [کّ2, Lemma 3.7].) Having proven the theorem, we deduce the following corollary.

Corollary 1.5. Suppose that the pair $(\mathfrak{g}, \mathfrak{s})$ satisfies the condition $(\mathbf{Q 1})$. Then both $\left(\mathfrak{g}, \mathfrak{g}_{1}\right)$ and $\left(\mathfrak{g}_{1}, \mathfrak{s}\right)$ satisfy $(\mathbf{Q 1})$ as well.

Proof. Let us first show that $\left(\mathfrak{g}_{1}, \mathfrak{s}\right)$ satisfies $(\mathbf{Q} 1)$. For that purpose let $\mathfrak{t}$ be any Cartan subalgebra of $\mathfrak{s}$. By the assumption of the corollary, there exists a unique Cartan subalgebra $\mathfrak{h}$ of $\mathfrak{g}$ so that $\mathfrak{t} \subseteq \mathfrak{h}$. Our theorem ensures that there exists $\vartheta \in \mathfrak{t}$ which is regular in $\mathfrak{g}$. By [D, Prop. 1.9.13], $\vartheta$ is also regular in $\mathfrak{g}_{1}$. Applying our theorem once more, but this time to the pair $\left(\mathfrak{g}_{1}, \mathfrak{s}\right)$, we conclude that there exists a unique Cartan subalgebra $\mathfrak{h}_{1}$ of $\mathfrak{g}_{1}$ such that $\mathfrak{t} \subseteq \mathfrak{h}_{1}$. It is worth noting that for the Cartan subalgebras $\mathfrak{t}, \mathfrak{h}_{1}$ and $\mathfrak{h}$ we have the following:

$$
\mathfrak{h}_{1}=C_{\mathfrak{g}_{1}}(\mathfrak{t}) \subseteq C_{\mathfrak{g}}(\mathfrak{t})=\mathfrak{h} .
$$

Let us now show that $\left(\mathfrak{g}, \mathfrak{g}_{1}\right)$ also satisfies $(\mathbf{Q 1})$. Suppose the contrary. Then we can find a Cartan subalgebra $\mathfrak{h}_{1}$ of $\mathfrak{g}_{1}$, and two different Cartan subalgebras $\mathfrak{h}, \mathfrak{h}^{\prime}$ of $\mathfrak{g}$, both containing $\mathfrak{h}_{1}$. Again, let $\mathfrak{t}$ be an arbitrary Cartan subalgebra of $\mathfrak{s}$. By the first claim of the corollary, there is a Cartan subalgebra $\mathfrak{h}_{1}^{\prime}$ of $\mathfrak{g}_{1}$ containing t. Denote by $\mathcal{G}_{1}$ the adjoint group of $\mathfrak{g}_{1}$, and take an element $g \in \mathcal{G}_{1}$ satisfying $g \cdot \mathfrak{h}_{1}^{\prime}=\mathfrak{h}_{1}$. Define a Lie algebra $\widetilde{\mathfrak{s}}=g \cdot \mathfrak{s}$. Clearly, $\widetilde{\mathfrak{s}}$ is reductive in $\mathfrak{g}_{1}$. Next define $\mathfrak{c}=g \cdot \mathfrak{t}$, which is a Cartan subalgebra of $\widetilde{\mathfrak{s}}$. Furthermore, both $\mathfrak{h}$ and $\mathfrak{h}^{\prime}$ contain $\mathfrak{c}$. Thus, the corollary follows by applying the following claim.

Claim. ( $\mathfrak{g}, \widetilde{\mathfrak{s}})$ satisfies $(\mathbf{Q 1})$.

For the Claim, suppose $\widetilde{\mathfrak{u}}$ is a Cartan subalgebra of $\widetilde{\mathfrak{s}}$ for which there are two different Cartan subalgebras $\mathfrak{v}, \mathfrak{v}^{\prime}$ of $\mathfrak{g}$ that both contain it. Put $\mathfrak{u}=g^{-1} \cdot \widetilde{\mathfrak{u}}, \mathfrak{k}=g^{-1} \cdot \mathfrak{v}$ and $\mathfrak{k}^{\prime}=g^{-1} \cdot \mathfrak{v}^{\prime}$, where $g$ is as above. Then for these Cartan subalgebras $\mathfrak{u}$ of $\mathfrak{s}$ and $\mathfrak{k}, \mathfrak{k}^{\prime}$ of $\mathfrak{g}$, we have $\mathfrak{k} \neq \mathfrak{k}^{\prime}$ and $\mathfrak{u} \subseteq \mathfrak{k}, \mathfrak{k}^{\prime}$. We conclude that $(\mathfrak{g}, \mathfrak{s})$ does not satisfy (Q1), a contradiction. 


\section{Cartan subspaces}

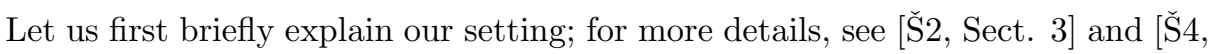
Sect. 2].

Suppose we have a pair of $\mathbb{K}$-Lie algebras $\left(\mathfrak{g}, \mathfrak{g}_{1}\right)$ so that $\mathfrak{g}_{1}$ is a proper subalgebra of $\mathfrak{g}$. In order to avoid certain trivialities we also assume that $\mathfrak{g}_{1}$ is not an ideal of $\mathfrak{g}$. We say that $\left(\mathfrak{g}, \mathfrak{g}_{1}\right)$ is a symmetric pair if there is some involutive automorphism $\theta$ of $\mathfrak{g}$ so that $\mathfrak{g}_{1}=\mathfrak{g}^{\theta}$; otherwise the pair is nonsymmetric.

For $\left(\mathfrak{g}, \mathfrak{g}_{1}\right)$, let $\boldsymbol{\beta}$ be the restriction of $B_{\mathfrak{g}}$ to $\mathfrak{g}_{1}$. The pairs we are interested in will always satisfy the following:

(C) The form $\boldsymbol{\beta}$ is nondegenerate.

Notice that although the previous condition is in a sense rather strong, it is not very restrictive. In other words, there are a number of pairs $\left(\mathfrak{g}, \mathfrak{g}_{1}\right)$ satisfying $(\mathbf{C})$; see Sections 1 and 2 of $\left[\underline{\mathrm{S}} 3\right.$ ]. Now take such a pair $\left(\mathfrak{g}, \mathfrak{g}_{1}\right)$. Let $r: \mathfrak{g}^{*} \rightarrow \mathfrak{g}_{1}^{*}$ be the restriction map between the duals of $\mathfrak{g}$ and $\mathfrak{g}_{1}$, and let $\kappa: \mathfrak{g} \rightarrow \mathfrak{g}^{*}$ be the Killing homomorphism. Define also an isomorphism $\kappa_{1}: \mathfrak{g}_{1} \rightarrow \mathfrak{g}_{1}^{*}$, given by $\kappa_{1}\left(x_{1}\right)=\boldsymbol{\beta}\left(x_{1},.\right)$, for $x_{1} \in \mathfrak{g}_{1}$. Let $\pi: \mathfrak{g} \rightarrow \mathfrak{g}_{1}$ be a linear map satisfying $\kappa_{1} \circ \pi=r \circ \kappa$. Define a subspace

$$
\mathfrak{p}=\operatorname{ker} \pi \text {. }
$$

Proposition 2.1. Let $\left(\mathfrak{g}, \mathfrak{g}_{1}\right)$ and $\mathfrak{p}$ be as above.

(i) We have a Killing-orthogonal direct sum decomposition

$$
\mathfrak{g}=\mathfrak{g}_{1} \oplus \mathfrak{p},
$$

where $\left[\mathfrak{g}_{1}, \mathfrak{p}\right] \subseteq \mathfrak{p}$.

(ii) The pair $\left(\mathfrak{g}, \mathfrak{g}_{1}\right)$ is symmetric if and only if $[\mathfrak{p}, \mathfrak{p}] \subseteq \mathfrak{g}_{1}$.

Recall the following well-known fact, in a form suitable for our purposes; see B, Ch. I, $\S 6.5$, Cor. 4].

Lemma 2.2. Let $\mathfrak{s}$ be a Lie algebra, $\mathfrak{u}$ an ideal of $\mathfrak{s}$ and $\rho$ a semisimple representation of $\mathfrak{s}$. Then the restriction of $\rho$ to $\mathfrak{u}$ is semisimple as well.

Now let $\mathfrak{g}$ be a semisimple Lie algebra. Let $\mathfrak{g}_{1}$ be a subalgebra reductive in $\mathfrak{g}$. Suppose that the pair $\left(\mathfrak{g}, \mathfrak{g}_{1}\right)$ satisfies the condition $(\mathbf{C})$; thus in particular we have the decomposition (2). Given a Cartan subalgebra $\mathfrak{h}_{1}$ of $\mathfrak{g}_{1}$, let $\mathfrak{h}$ be any Cartan subalgebra of $\mathfrak{g}$ containing $\mathfrak{h}_{1}$. Define the "vector part", corresponding to $\mathfrak{h}$, by

$$
\mathfrak{a}_{\mathfrak{h}}=\mathfrak{h} \cap \mathfrak{p}
$$

(cf. [BK, Eq. (5-5)]. Notice here this simple fact; see [Š2, Lemma 3.9(3)].

Lemma 2.3. We have a direct sum decomposition

$$
\mathfrak{h}=\mathfrak{h}_{1} \oplus \mathfrak{a}_{\mathfrak{h}} .
$$

When $\mathfrak{g}$ is semisimple symmetric, the following definition is classical.

Definition 2.4. Let $\left(\mathfrak{g}, \mathfrak{g}_{1}\right)$ be as above, and so (2) holds. A Cartan subspace of $\mathfrak{g}$ (or a Cartan subspace of $\mathfrak{p}$ ) is a commutative subalgebra $\mathfrak{a} \subseteq \mathfrak{p}$ of $\mathfrak{g}$, which is reductive in $\mathfrak{g}$ and satisfies $C_{\mathfrak{p}}(\mathfrak{a})=\mathfrak{a}$.

A standard argument gives the following basic statement.

Lemma 2.5. Let $\left(\mathfrak{g}, \mathfrak{g}_{1}\right)$ be a pair of $\mathbb{K}$-Lie algebras, and $\mathbb{F} / \mathbb{K}$ a field extension. Then $\mathfrak{a}$ is a Cartan subspace of $\mathfrak{g}$ if and only if $\mathfrak{a}^{\mathbb{F}}$ is a Cartan subspace of $\mathfrak{g}^{\mathbb{F}}$. 
Now we are going to specify certain interesting pairs of Lie algebras that we will study.

Definition 2.6. Let $\left(\mathfrak{g}, \mathfrak{g}_{1}\right)$ be a pair satisfying both $(\mathbf{C})$ and $(\mathbf{Q 1})$. We say that it is a Cartan pair if the following two conditions hold:

(Cp1) There exist a Cartan subspace $\mathfrak{a}$ and a Cartan subalgebra $\mathfrak{h}$, of $\mathfrak{g}$, such that

$$
\left(\mathfrak{h} \cap \mathfrak{g}_{1}\right) \oplus \mathfrak{a}=\mathfrak{h} .
$$

(Cp2) If we put

$$
\Delta^{\prime}=\left\{\phi \in \Delta(\overline{\mathfrak{g}}, \overline{\mathfrak{h}}) \mid \phi_{\mid \overline{\mathfrak{a}}}=0\right\}
$$

then

$$
\phi \in \Delta^{\prime} \Rightarrow X_{\phi} \in \overline{\mathfrak{g}_{1}} .
$$

Our first useful observations about Cartan pairs are contained in the following proposition; cf. [ัS1, Thm. 2.7(ii)] with the claim (i). Note that it is a generalization of [D, Prop. 1.13.7]; see Proposition 2.13 below.

Proposition 2.7. Suppose that $\left(\mathfrak{g}, \mathfrak{g}_{1}\right)$ is a Cartan pair, and let $\mathfrak{h}$ and $\mathfrak{a}$ be as in

(Cp1). Denote by $\mathfrak{m}$ the centralizer $C_{\mathfrak{g}_{1}}(\mathfrak{a})$.

(i) We have a direct sum decomposition

$$
C_{\mathfrak{g}}(\mathfrak{a})=\mathfrak{m} \oplus \mathfrak{a},
$$

and (cf. (3) $)$

$$
\mathfrak{h} \cap \mathfrak{p}=\mathfrak{a} .
$$

(ii) The restrictions of $B_{\mathfrak{g}}$ to $\mathfrak{a}$ and $\mathfrak{m}$ are nondegenerate.

(iii) The Lie algebra $\mathfrak{m}$ is reductive in $\mathfrak{g}$.

(iv) Let $\mathfrak{l}_{1}$ be any Cartan subalgebra of $\mathfrak{m}$. Define $\mathfrak{l}=\mathfrak{l}_{1} \oplus \mathfrak{a}$. Then $\mathfrak{l}$ is a Cartan subalgebra of $\mathfrak{g}$.

Proof. By Lemma 2.5 we may assume that $\mathbb{K}$ is algebraically closed.

(i) Take any $x \in C_{\mathfrak{g}}(\mathfrak{a})$. By putting

$$
\Delta^{\prime \prime}=\left\{\phi \in \Delta(\mathfrak{g}, \mathfrak{h}) \mid \phi_{\mid \mathfrak{a}} \neq 0\right\},
$$

we can write

$$
x=\sum_{\Delta^{\prime}} c_{\phi} X_{\phi}+\sum_{\Delta^{\prime \prime}} c_{\phi} X_{\phi}+\vartheta_{1}+\alpha
$$

where $c_{\phi} \in \mathbb{K}, \vartheta_{1} \in \mathfrak{h} \cap \mathfrak{g}_{1}$ and $\alpha \in \mathfrak{a}$. Let $a \in \mathfrak{a}$ be such that $\phi(a) \neq 0$, for all $\phi \in \Delta^{\prime \prime}$. Using that $[a, x]=0$, we deduce at once that $c_{\phi}=0$, for all $\phi \in \Delta^{\prime \prime}$. Also, $\left[\vartheta_{1}, \mathfrak{a}\right]=0$ implies that in particular $\vartheta_{1} \in C_{\mathfrak{g}_{1}}(\mathfrak{a})$. So we have $x=y+\vartheta_{1}+\alpha$, where $y=\sum_{\Delta^{\prime}} c_{\phi} X_{\phi}$. By $(\mathbf{C p} 2)$, we have that $y \in \mathfrak{g}_{1}$. Furthermore, $y \in C_{\mathfrak{g}_{1}}(\mathfrak{a})$. Thus we have proved the inclusion from left to right in (5), and the opposite inclusion is obvious.

Take any $x \in \mathfrak{h} \cap \mathfrak{p}$, and write it as $x=\vartheta_{1}+\alpha$, in accordance with (4). Hence it is immediate that $\vartheta_{1}=x-\alpha=0$; i.e., $x \in \mathfrak{a}$. So we have proved the inclusion $\mathfrak{h} \cap \mathfrak{p} \subseteq \mathfrak{a}$; the opposite inclusion is clear.

Now the claims (ii), (iii) and (iv) have the same proofs as in the symmetric case; see the corresponding claims in [D, Prop. 1.13.7]. 
Again let $\left(\mathfrak{g}, \mathfrak{g}_{1}\right)$ be a Cartan pair, and let $\mathfrak{h}$ and $\mathfrak{a}$ be as in (Cp1). Suppose furthermore that $\mathfrak{h}$ is splitting. For $\lambda \in \mathfrak{a}^{*}$ define a subspace

$$
\mathfrak{g}_{\lambda}=\{x \in \mathfrak{g} \mid[H, x]=\lambda(H) x \text { for all } H \in \mathfrak{a}\},
$$

and then

$$
\Sigma=\Sigma(\mathfrak{g}, \mathfrak{a})=\left\{\lambda \in \mathfrak{a}^{*} \mid \mathfrak{g}_{\lambda} \neq 0\right\} .
$$

Now we can state the next proposition which provides some further information concerning Cartan pairs.

Proposition 2.8. Let $\left(\mathfrak{g}, \mathfrak{g}_{1}\right), \mathfrak{h}$ and $\mathfrak{a}$ be as above.

(i) We have a direct sum decomposition

$$
\mathfrak{g}=\mathfrak{g}_{0} \oplus \bigoplus_{\Sigma} \mathfrak{g}_{\lambda}
$$

(ii) We have $\left[\mathfrak{g}_{\lambda}, \mathfrak{g}_{\mu}\right] \subseteq \mathfrak{g}_{\lambda+\mu}$, for $\lambda, \mu \in \mathfrak{a}^{*}$.

(iii) If $\lambda \in \Sigma$, then $-\lambda \in \Sigma$.

(iv) If $\lambda, \mu \in \mathfrak{a}^{*}$ are such that $\lambda+\mu \neq 0$, then $B_{\mathfrak{g}}\left(\mathfrak{g}_{\lambda}, \mathfrak{g}_{\mu}\right)=0$. In particular, the restriction of $B_{\mathfrak{g}}$ to $\mathfrak{g}_{\lambda} \times \mathfrak{g}_{-\lambda}$ is nondegenerate.

Proof. As $\{\operatorname{ad} H \mid H \in \mathfrak{a}\}$ is a commuting family of diagonizable operators, (i) is immediate. For (ii) one just has to use the Jacobi identity.

(iii) First define a map

$$
\Psi=\Psi_{\mathfrak{h}, \mathfrak{a}}: \mathfrak{g} \rightarrow \mathfrak{g}
$$

as follows. Given any $x \in \mathfrak{g}$, write it as $x=\sum_{\Delta} c_{\phi} X_{\phi}+\vartheta$, where $\Delta=\Delta(\mathfrak{g}, \mathfrak{h})$, $\vartheta \in \mathfrak{h}$ and $c_{\phi} \in \mathbb{K}$. Then put

$$
\Psi(x)=\sum_{\Delta} c_{\phi} X_{-\phi}+\vartheta
$$

Now let $x \in \mathfrak{g}_{\lambda}$ be nonzero, and write it as above. Then obviously $\vartheta=0$ and $c_{\phi} \phi(H)=c_{\phi} \lambda(H)$, for all $\phi \in \Delta$ and $H \in \mathfrak{a}$. Hence it follows at once that $\Psi(x) \in \mathfrak{g}_{-\lambda}$.

(iv) Take $a \in \mathfrak{a}$ so that $(\lambda+\mu)(a) \neq 0$. Using the invariance of $B_{\mathfrak{g}}$, we have that

$$
0=B_{\mathfrak{g}}\left(\left[a, \mathfrak{g}_{\lambda}\right], \mathfrak{g}_{\mu}\right)+B_{\mathfrak{g}}\left(\mathfrak{g}_{\lambda},\left[a, \mathfrak{g}_{\mu}\right]\right)=(\lambda+\mu)(a) B_{\mathfrak{g}}\left(\mathfrak{g}_{\lambda}, \mathfrak{g}_{\mu}\right),
$$

and therefore $B_{\mathfrak{g}}\left(\mathfrak{g}_{\lambda}, \mathfrak{g}_{\mu}\right)=0$. By the nondegeneracy of the form $B_{\mathfrak{g}}$, it follows that its restriction to $\mathfrak{g}_{\lambda} \times \mathfrak{g}_{-\lambda}$ is nondegenerate as well; here we use (iii).

Remark 2.9. Let $\left(\mathfrak{g}, \mathfrak{g}_{1}\right)$ and the map $\pi$ be as in the paragraph preceding Proposition 2.1. Put $\theta=2 \pi-1_{\mathfrak{g}}$; i.e., for $x=x_{1}+p$, where $x_{1} \in \mathfrak{g}_{1}$ and $p \in \mathfrak{p}$, we have $\theta(x)=x_{1}-p$. Of course, $\left(\mathfrak{g}, \mathfrak{g}_{1}\right)$ is a symmetric pair if and only if $\theta$ is an automorphism of $\mathfrak{g}$, and then $\mathfrak{g}_{1}=\mathfrak{g}^{\theta}$. In that case we have $[H, \theta(x)]=-\lambda(H) \theta(x)$, where $H \in \mathfrak{a}, \lambda \in \Sigma$ and $x \in \mathfrak{g}_{\lambda}$. As a consequence, for such an $x$ it follows that $\theta(x) \in \mathfrak{g}_{-\lambda}$; cf. [Kn, Prop. 6.40]. Concerning (iii) of the previous proposition, let us emphasize that for $\left(\mathfrak{g}, \mathfrak{g}_{1}\right)$ nonsymmetric we cannot expect to have the same as above. More precisely, it can happen that for nonzero $x=x_{1}+p \in \mathfrak{g}_{\lambda}$ as before, we cannot find a nonzero $y \in \operatorname{span}_{\mathbb{K}}\left\{x_{1}, p\right\}$ satisfying $y \in \mathfrak{g}_{-\lambda}$. For an example showing this exists for the nonsymmetric pair of type $\left(B_{3}, G_{2}\right)$, see Example 2.14. We leave the details to the reader.

The next easy lemma points at a subclass of Cartan pairs that seems to be simpler for studying. 
Lemma 2.10. Let $\left(\mathfrak{g}, \mathfrak{g}_{1}\right)$ be a pair satisfying $(\mathbf{C})$ and the condition on ranks $\mathrm{rk} \mathfrak{g}_{1}<$ rk $\mathfrak{g}$. Suppose that there exists a pair of the corresponding Cartan subalgebras $\mathfrak{h}_{1} \subseteq$ $\mathfrak{h}$, and then let $\mathfrak{a}=\mathfrak{a}_{\mathfrak{h}}$ be as in (3). Suppose also that the condition (Cp2) holds. Then $\mathfrak{a}$ is a Cartan subspace of $\mathfrak{g}$, and $\mathfrak{h} \cap \mathfrak{g}_{1}=\mathfrak{h}_{1}$. As a consequence we have that if the pair $\left(\mathfrak{g}, \mathfrak{g}_{1}\right)$ moreover satisfies $(\mathbf{Q} \mathbf{1})$, then it is a Cartan pair.

Proof. Here we will again assume that $\mathbb{K}$ is algebraically closed.

To prove that $\mathfrak{h} \cap \mathfrak{g}_{1}=\mathfrak{h}_{1}$ we proceed in the same way as for (6). Next, by Lemmas 2.2 and 2.3 , we have that $\mathfrak{a} \subseteq \mathfrak{p}$ is commutative and reductive in $\mathfrak{g}$. Therefore, to prove that $\mathfrak{a}$ is a Cartan subspace, it remains to show the inclusion $C_{\mathfrak{p}}(\mathfrak{a}) \subseteq \mathfrak{a}$. For that purpose, let $x \in C_{\mathfrak{p}}(\mathfrak{a})$ be arbitrary. Having $\Delta^{\prime}$ and $\Delta^{\prime \prime}$ as above, write $x$ as in (7); here we use Lemma 2.3 again. The same argument as in Proposition 2.7 gives that now $x=\alpha \in \mathfrak{a}$.

By the last lemma, it is clear that we can refine our definition of Cartan pairs as follows:

Definition 2.11. A Cartan pair $\left(\mathfrak{g}, \mathfrak{g}_{1}\right)$ will be called a Cartan pair of type $I$ if we have $r k \mathfrak{g}_{1}<\mathrm{rk} \mathfrak{g}$, and for $\mathfrak{a}$ and $\mathfrak{h}$ as in $(\mathbf{C p} \mathbf{1})$ there exists a Cartan subalgebra $\mathfrak{h}_{1}$ of $\mathfrak{g}_{1}$, contained in $\mathfrak{h}$, so that $\mathfrak{a}$ is given as in (3). If $\left(\mathfrak{g}, \mathfrak{g}_{1}\right)$ is not of type I, we will say that it is a Cartan pair of type II.

The corollary given below is in fact [ک̌1, Thm. 2.7(i)].

Corollary 2.12. Assume that $\mathbb{K}$ is algebraically closed. Then the definition of Cartan pairs of type $I$ does not depend on the choice of a Cartan subalgebra of $\mathfrak{g}_{1}$. More precisely, suppose that $\mathfrak{h}_{1}$ and $\mathfrak{c}_{1}$ are two Cartan subalgebras of $\mathfrak{g}_{1}$, and then let $\mathfrak{h}$ ad $\mathfrak{c}$ be the (unique) Cartan subalgebras of $\mathfrak{g}$ so that $\mathfrak{h}_{1} \subseteq \mathfrak{h}$ and $\mathfrak{c}_{1} \subseteq \mathfrak{c}$. Let $\mathfrak{a}_{\mathfrak{h}}$ and $\mathfrak{a}_{\mathfrak{c}}$ be the corresponding subspaces of $\mathfrak{p}$. Then (Cp2) holds, with $\mathfrak{h}$ and $\mathfrak{a}=\mathfrak{a}_{\mathfrak{h}}$, if and only if it holds with $\mathfrak{h}$ replaced by $\mathfrak{c}$ and $\mathfrak{a}=\mathfrak{a}_{\mathfrak{c}}$.

Now we will show that when we focus on Cartan subspaces, our notion of Cartan pair generalizes the notion of symmetric pair. Moreover, we characterize those symmetric pairs that are of type I.

Proposition 2.13. Let $\left(\mathfrak{g}, \mathfrak{g}_{1}\right)$ be a symmetric pair of Lie algebras. Then it is a Cartan pair.

Suppose that $\mathrm{rk} \mathfrak{g}_{1}<\mathrm{rk} \mathfrak{g}$. Then the following are equivalent:

(a) There exists a Cartan subalgebra $\mathfrak{h}_{1}$ of $\mathfrak{g}_{1}$ such that $\mathfrak{a}=C_{\mathfrak{g}}\left(\mathfrak{h}_{1}\right) \cap \mathfrak{p}$ is a Cartan subspace of $\mathfrak{g}$.

(b) For every Cartan subspace $\mathfrak{a}$ of $\mathfrak{g}$ we have the equality

$$
\operatorname{rkg} \mathfrak{g}_{1}=\operatorname{rk} C_{\mathfrak{g}_{1}}(\mathfrak{a}) .
$$

(c) There exists a Cartan subspace $\mathfrak{a}$ of $\mathfrak{g}$ for which we have (8).

(d) The Cartan pair $\left(\mathfrak{g}, \mathfrak{g}_{1}\right)$ is of type $I$.

Provided that $\mathbb{K}$ is algebraically closed, the above four statements are further equivalent to the following:

(e) For every Cartan subalgebra $\mathfrak{h}_{1}$ of $\mathfrak{g}_{1}$, the set $\mathfrak{a}=C_{\mathfrak{g}}\left(\mathfrak{h}_{1}\right) \cap \mathfrak{p}$ is a Cartan subspace of $\mathfrak{g}$. 
Proof. For a symmetric pair it is well known that (C) holds. We have (Q1) as well; for a more general result, see [S̆2, Cor. 4.5]. Having the usual decomposition $\mathfrak{g}=\mathfrak{g}_{1} \oplus \mathfrak{p}$, there exists a Cartan subspace $\mathfrak{a} \subseteq \mathfrak{p}$ of $\mathfrak{g}$. Furthermore, for a Cartan subalgebra $\mathfrak{l}$ of $C_{\mathfrak{g}_{1}}(\mathfrak{a})$, the subspace $\mathfrak{h}=\mathfrak{l} \oplus \mathfrak{a}$ is a Cartan subalgebra of $\mathfrak{g}$. Clearly, $\mathfrak{h} \cap \mathfrak{g}_{1}=\mathfrak{l}$. Thus we have (Cp1) of Definition 2.6. In order to see that (Cp2) holds as well, one just has to take into account D, Prop. 1.13.9(iii)].

Let us now show that the above statements are mutually equivalent. First note that the implications (b) $\Rightarrow(\mathrm{c}),(\mathrm{d}) \Rightarrow(\mathrm{a})$ and $(\mathrm{e}) \Rightarrow(\mathrm{a})$ are trivial.

Suppose that (a) holds, and let $\mathfrak{h}_{1}$ be as there. Also let $\mathfrak{a}^{\prime}$ be an arbitrary Cartan subspace of $\mathfrak{g}$. Define $\mathfrak{m}^{\prime}=C_{\mathfrak{g}_{1}}\left(\mathfrak{a}^{\prime}\right)$. Let $\mathfrak{l}^{\prime}$ be any Cartan subalgebra of $\mathfrak{m}^{\prime}$; then $\mathfrak{l}^{\prime} \oplus \mathfrak{a}^{\prime}$ is a Cartan subalgebra of $\mathfrak{g}$. On the other hand, for the Cartan subalgebra $\mathfrak{h}=C_{\mathfrak{g}}\left(\mathfrak{h}_{1}\right)$ we have that $\mathfrak{a}=\mathfrak{a}_{\mathfrak{h}}=\mathfrak{h} \cap \mathfrak{p}$ is a Cartan subspace of $\mathfrak{g}$. Furthermore, by Lemma 2.3. we know that $\mathfrak{h}_{1} \oplus \mathfrak{a}=\mathfrak{h}$. As $\mathfrak{a}$ and $\mathfrak{a}^{\prime}$ are of the same rank, we deduce that $\operatorname{dim} \mathfrak{l}^{\prime}=\operatorname{dim} \mathfrak{h}_{1}$; i.e., $\operatorname{rk} \mathfrak{m}^{\prime}=\operatorname{rk} \mathfrak{g}_{1}$. Thus we have proved that (b) holds.

Now suppose (c) holds, but let us write $\mathfrak{a}^{\prime}$ instead of $\mathfrak{a}$. We know that $\left(\mathfrak{g}, \mathfrak{g}_{1}\right)$ is a Cartan pair, and so it satisfies the two conditions of Definition 2.6. Let $\mathfrak{a}$ and $\mathfrak{h}$ be as there. We have to show that there is a Cartan subalgebra $\mathfrak{h}_{1}$ of $\mathfrak{g}_{1}$ such that $\mathfrak{h}_{1} \subseteq \mathfrak{h}$. To see this, first note that

$$
\operatorname{rk} \mathfrak{m}=\operatorname{rk} \mathfrak{g}-\operatorname{rk} \mathfrak{a},
$$

where $\mathfrak{m}=C_{\mathfrak{g}_{1}}(\mathfrak{a})$. At the same time we have

$$
\operatorname{rk} \mathfrak{g}_{1}=\mathrm{rk}^{\prime} \mathfrak{m}^{\prime}=\mathrm{rkg}-\operatorname{rk} \mathfrak{a}^{\prime},
$$

where $\mathfrak{m}^{\prime}=C_{\mathfrak{g}_{1}}\left(\mathfrak{a}^{\prime}\right)$. It follows that $\operatorname{rk} \mathfrak{g}_{1}=$ rk $\mathfrak{m}$. Now define $\mathfrak{h}_{1}=\mathfrak{h} \cap \mathfrak{g}_{1}$. Clearly, by the condition (Cp1) and (9), we have that $\mathfrak{h}_{1} \subseteq \mathfrak{m}$ and $\operatorname{dim} \mathfrak{h}_{1}=$ rk $\mathfrak{m}$. Thus, $\mathfrak{h}_{1}$ is a Cartan subalgebra of $\mathfrak{m}$, and so it is a Cartan subalgebra of $\mathfrak{g}_{1}$ as well, what we had to show.

Suppose now that $\mathbb{K}$ is algebraically closed, and that (a)-(d) hold. Let $\mathfrak{h}_{1}^{\prime}$ be an arbitrary Cartan subalgebra of $\mathfrak{g}_{1}$. Then $\mathfrak{h}^{\prime}=C_{\mathfrak{g}}\left(\mathfrak{h}_{1}^{\prime}\right)$ is a (unique) Cartan subalgebra of $\mathfrak{g}$ containing $\mathfrak{h}_{1}^{\prime}$. Define $\mathfrak{a}^{\prime}=\mathfrak{h}^{\prime} \cap \mathfrak{p}$. We will show that it is a Cartan subspace of $\mathfrak{g}$. For that, take $\mathfrak{a}$ and $\mathfrak{h}$ to be as in Definition 2.6. As $\left(\mathfrak{g}, \mathfrak{g}_{1}\right)$ is of type I, there is a Cartan subalgebra $\mathfrak{h}_{1}$ of $\mathfrak{g}_{1}$ such that $\mathfrak{h}_{1} \subseteq \mathfrak{h}$. For the adjoint group $\mathcal{G}_{1}$ of $\mathfrak{g}_{1}$, let $g \in \mathcal{G}_{1}$ be such that $g \cdot \mathfrak{h}_{1}=\mathfrak{h}_{1}^{\prime}$. As $g \cdot \mathfrak{h}$ is a Cartan subalgebra of $\mathfrak{g}$ containing $\mathfrak{h}_{1}^{\prime}$, it follows that $g \cdot \mathfrak{h}=\mathfrak{h}^{\prime}$. Furthermore, we have $g \cdot \mathfrak{p}=\mathfrak{p}$, and as an immediate consequence that $g \cdot \mathfrak{a}=\mathfrak{a}^{\prime}$. This shows that $\mathfrak{a}^{\prime}$ is a Cartan subspace of $\mathfrak{g}$.

In order to see what we could expect to have in more general situations, let us close the paper by recalling in detail one example; see [ک1, Sect. 3]. Notice that the notation in the cited work is different than here.

Example 2.14. Let $\mathfrak{s}=\mathfrak{s o}(8, \mathbb{K})$, standardly embedded in $\mathfrak{s l}(8, \mathbb{K})$. Put $H_{i}=$ $E_{i i}-E_{9-i, 9-i}$, for $1 \leq i \leq 4$. Then $\mathfrak{t}=\operatorname{span}_{\mathbb{K}}\left\{H_{1}, \ldots, H_{4}\right\}$ is a split Cartan subalgebra of $\mathfrak{s}$. Let $\left(\epsilon_{i}\right)$ be the dual basis of $\left(H_{i}\right)$. Define $\mathfrak{h}=\operatorname{span}_{\mathbb{K}}\left\{H_{1}, H_{2}, H_{3}\right\}$, and the restrictions $\eta_{i}=\epsilon_{i \mid \mathfrak{h}}$. Put

$$
\Delta^{+}=\left\{\eta_{1}, \eta_{2}, \eta_{3}\right\} \cup\left\{\eta_{i} \pm \eta_{j} \mid 1 \leq i<j \leq 3\right\},
$$

and $\Delta=\Delta^{+} \cup\left(-\Delta^{+}\right)$. For $1 \leq i \leq 3$ define

$$
X_{\eta_{i}}=2 E_{i 4}+E_{4,9-i}, \quad X_{-\eta_{i}}=-2 E_{9-i, 4}-E_{4 i},
$$


and for $1 \leq i<j \leq 3$ define

$$
\begin{array}{ll}
X_{\eta_{i}-\eta_{j}}=E_{i j}-E_{9-j, 9-i}, & X_{-\eta_{i}+\eta_{j}}=-E_{j i}+E_{9-i, 9-j}, \\
X_{\eta_{i}+\eta_{j}}=E_{i, 9-j}-E_{j, 9-i}, & X_{-\eta_{i}-\eta_{j}}=-E_{9-j, i}+E_{9-i, j} .
\end{array}
$$

Then

$$
\mathfrak{g}=\mathfrak{h} \oplus \bigoplus_{\phi \in \Delta} \mathbb{K} X_{\phi}
$$

is a simple Lie algebra of type $B_{3}$; i.e., $\mathfrak{g}=\mathfrak{s o}(7, \mathbb{K})$. Also let $\Pi=\left\{\beta_{1}, \beta_{2}, \beta_{3}\right\}$, where $\beta_{1}=\eta_{1}-\eta_{2}, \beta_{2}=\eta_{2}-\eta_{3}$ and $\beta_{3}=\eta_{3}$. Then $\Pi$ is a basis of the root system $\Delta$.

Consider the Dynkin diagram of type $D_{4}$, labeled as in $\mathrm{LS}$. Let $\sigma$ be its order 3 automorphism satisfying $\sigma\left(\epsilon_{3}+\epsilon_{4}\right)=\epsilon_{3}-\epsilon_{4}$. Lift this $\sigma$ (uniquely) to an automorphism of $\mathfrak{s}$. Let $\mathfrak{g}_{1}=\mathfrak{s}^{\sigma}$ be the fixed point algebra for $\sigma$. Then $\mathfrak{g}_{1}$ is a simple Lie algebra of type $G_{2}$, and $\mathfrak{h}_{1}=\mathfrak{g}_{1} \cap \mathfrak{t}$ is its split Cartan subalgebra. Choose a basis $\Pi_{1}=\left\{\alpha_{1}, \alpha_{2}\right\}$ of the root system $\Delta_{1}=\Delta\left(\mathfrak{g}_{1}, \mathfrak{h}_{1}\right)$, so that the Chevalley basis for $\mathfrak{g}_{1}$ in terms of that for $\mathfrak{g}$ is given as in [Š1, Sect. 3.3]; cf. [LS, Sect. 2.3]. Notice that the root $\alpha_{1}$ is short, while $\alpha_{2}$ is long.

For the pair $\left(\mathfrak{g}, \mathfrak{g}_{1}\right)$, let $\mathfrak{g}=\mathfrak{g}_{1} \oplus \mathfrak{p}$ be the usual decomposition. Define the one-dimensional subspace $\mathfrak{a} \subseteq \mathfrak{p}$ by

$$
\mathfrak{a}=\mathbb{K a}, \quad \text { for } \mathrm{a}=-H_{1}+H_{2}+H_{3} .
$$

Fact. The pair $\left(\mathfrak{g}, \mathfrak{g}_{1}\right)$ is a nonsymmetric Cartan pair of type $\mathrm{I}$, and $\mathfrak{a}$ is its Cartan subspace. More precisely, this pair satisfies Definition 2.6, where $\mathfrak{a}$ and $\mathfrak{h}$ are as above.

Furthermore, it will be instructive to observe one more fact. First define $\lambda \in \mathfrak{a}^{*}$ by $\lambda(\mathrm{a})=1$. Then

$$
\Sigma=\Sigma(\mathfrak{g}, \mathfrak{a})=\{ \pm \lambda, \pm 2 \lambda\}
$$

and

$$
\begin{aligned}
\mathfrak{g}_{\lambda} & =\operatorname{span}_{\mathbb{K}}\left\{X_{\beta_{3}}, X_{\beta_{2}+\beta_{3}}, X_{-\beta_{1}-\beta_{2}-\beta_{3}}\right\}, \\
\mathfrak{g}_{-\lambda} & =\operatorname{span}_{\mathbb{K}}\left\{X_{-\beta_{3}}, X_{-\beta_{2}-\beta_{3}}, X_{\beta_{1}+\beta_{2}+\beta_{3}}\right\}, \\
\mathfrak{g}_{2 \lambda} & =\operatorname{span}_{\mathbb{K}}\left\{X_{-\beta_{1}}, X_{-\beta_{1}-\beta_{2}}, X_{\beta_{2}+2 \beta_{3}}\right\}, \\
\mathfrak{g}_{-2 \lambda} & =\operatorname{span}_{\mathbb{K}}\left\{X_{\beta_{1}}, X_{\beta_{1}+\beta_{2}}, X_{-\beta_{2}-2 \beta_{3}}\right\} .
\end{aligned}
$$

Define $\mathfrak{n}=\mathfrak{g}_{\lambda}+\mathfrak{g}_{2 \lambda}$. Then we have a direct sum decomposition

$$
\mathfrak{g}=\mathfrak{g}_{1} \oplus \mathfrak{a} \oplus \mathfrak{n},
$$

which is an analogue of the classical Iwasawa decomposition for symmetric pairs.

\section{ACKNOWLEDGMENTS}

The first version of this paper was written during the author's stay (April/May 2010) at the Department of Mathematics, University of Utah. The author is grateful to Dragan Miličić and Peter Trapa, who made possible this highly enjoyable visit. He is also grateful both to them and to Dan Ciubotaru for several very inspiring conversations. In particular, Dragan Miličić helped very much. Without his criticism and hints, this paper would not exist in its present form. 


\section{REFERENCES}

[B] N. Bourbaki, Lie groups and Lie algebras, Chapters 1-3, Springer-Verlag, Berlin, 1989. MR.979493 (89k:17001)

[BK] R. Brylinski and B. Kostant, Nilpotent orbits, normality, and Hamiltonian group actions, J. Amer. Math. Soc. 7 (1994), 269-298. MR1239505 (94g:22031)

[D] J. Dixmier, Enveloping Algebras, Graduate Studies in Mathematics, Vol. 11, Amer. Math. Soc., 1996. MR.1393197 (97c:17010)

[Kn] A. W. Knapp, Lie Groups beyond an Introduction, Second edition, Progress in Math., vol. 140, Birkhäuser, Boston-Basel-Berlin, 2002. MR.1920389 (2003c:22001)

[Ks] B. Kostant, The principal three-dimensional subgroup and the Betti numbers of a complex simple Lie group, Amer. J. Math. 81 (1959), 973-1032. MR0114875 (22:5693)

[LM] J. Lepowsky and G. W. McCollum, Cartan subspaces of symmetric Lie algebras, Trans. Amer. Math. Soc. 216 (1976), 217-228. MR0404361 (53:8163)

[LS] T. Levasseur and S. P. Smith, Primitive ideals and nilpotent orbits in type $G_{2}$, J. Algebra 114 (1988), 81-105. MR931902 (89f:17013)

[Š1] B. Širola, Some structural results for nonsymmetric pairs of Lie algebras, J. Algebra 185 (1996), 571-582. MR 1417386(97h:17031)

[Š2] _ Pairs of semisimple Lie algebras and their maximal reductive subalgebras, Algebr. Represent. Theory 11 (2008), 233-250. MR2403292(2009d:17017)

[Š3] _ Pairs of Lie algebras and their self-normalizing reductive subalgebras, J. Lie Theory 19 (2009), 735-766. MR2599002 (2011i:17016)

[క̆4] _ Normalizers and self-normalizing subgroups, Glas. Mat. Ser. III 46 (2011), 385-414. $\operatorname{MR} 2855021$

[Š5] , Normalizers, distinguished nilpotent orbits and compatible pairs of Borel subalgebras, in preparation.

[V1] D. A. Vogan, Jr., The orbit method and primitive ideals for semisimple Lie algebras, Lie Algebras and Related Topics, CMS Conf. Proc., vol. 5, Amer. Math. Soc., Providence, RI, 1986, pp. 281-316. MR832204 (87k:17015)

[V2] - Noncommutative algebras and unitary representations, Proc. Sympos. Pure Math., vol. 48, Amer. Math. Soc., Providence, RI, 1988, pp. 35-60. MR974331 (90a:22015)

Department of Mathematics, University of Zagreb, BijeničKa 30, 10000 Zagreb, Crohtia

E-mail address: sirola@math.hr 